
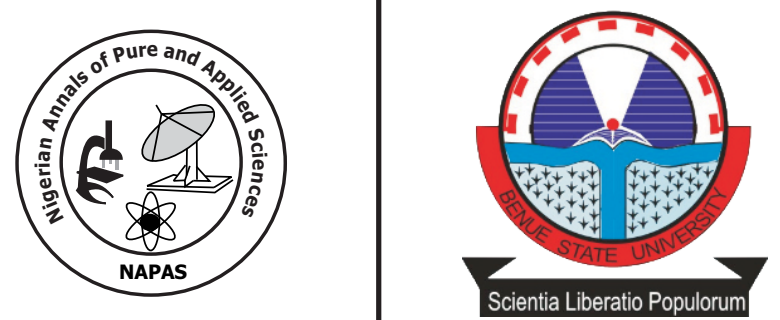

\title{
Mathematical Model of Geophysical Fluid Flow over Variable Bottom Topography
}

\author{
Iornumbe, S.I., Mbah G. C. E.; and Chia,R .A \\ Department of Mathematics and Computer Science, \\ Benue State University, Makurdi \\ iorsteve@yahoo.com
}

\begin{abstract}
In this paper, the bottom topography of a geophysical fluid flow is modelled in the presence of Coriolis force by the nonlinear shallow water equations. These equations, which are a system of three partial differential equations in two space dimensions, are solved using the perturbation method. The Effects of the Coriolis force and the bottom topography for particular initial flows on the velocity components and different kind of flow patterns possible in geophysical fluid flow have been studied and the results illustrated graphically.
\end{abstract}

Key words: Shallow Water Equations, Coriolis force, Series Solution, Bottom Topography 


\section{Introduction}

Shallow water equations are a set of hyperbolic partial differential equations that describe the flow below the pressure surface in the fluid, sometimes, but not necessarily, a free surface. The equations are derived from the principle of conservation of mass and momentum in the case where the horizontal length scale is much greater than the vertical length scale. They can be used to model waves in the atmosphere, rivers, lakes and oceans in a large domain as well as gravity waves. The rotating shallow water equations including topographic effects are a leading order model to study coastal hydrodynamics on several scales including intermediate scale rotational waves and breaking waves on beaches. Also, they are used with Coriolis forces in atmospheric and large water bodies' model as simplification of the primitive equations of the flow.

Due to the nonlinearity of the model as well as the complexity of the geometries encountered in real-life applications such as tsunamis, dam break and flooding, much effort has been made in recent years to develop numerical methods to solve the equations approximately.

Bottom topography plays a major role in determining the flow field in the large water bodies like oceans, rivers, and so on. One of the most important applications of the shallow water waves is the tsunami waves usually generated by underwater earthquakes which cause an irregular topography of increasing or decreasing water depth. In particular, the main problem in solving the shallow water equations is the presence of the source terms modeling the bottom topography and the Coriolis forces included in the system, (Dritschel et al., 1999).

The geophysical fluid flow of shallow water are based on the assumption that $\frac{H}{L}<<1$ where $H$ and $L$ are the characteristic values for the vertical and horizontal length scales of fluid respectively. These equations are a twodimensional hyperbolic system modeling the horizontal velocities for an incompressible fluid. Rotation is one of the most important factors that distinguishing geophysical fluid flow from classical fluid flow. If latitudinal varying Coriolis forces are includeding the shallow water equations, the resulting system can support gravity waves. The Coriolis force is proportional in magnitude to the flow speed and directed perpendicular to the direction of the flow. For a given horizontal motion, the strongest horizontal deflection is noted to be at the poles and there is no horizontal deflection at the equator and for vertical motion, the opposite is true. The magnitude of the Coriolis force proportionally depends upon the latitude and the wind speed. The direction of the Coriolis force always acts at right angles to the direction of motion, which is to the right in the Northern Hemisphere and to the left in the Southern Hemisphere (Pedlosky 1987).

A new well balance definite volume method within the framework of the finite volume evolution, Galerkin (FVEG) schemes for the shallow water equations with source terms modeling the Coriolis forces was presented (Lukácová-Medvid'ová et al., 2007).Also studied in Gallouët et al. (2003) was the computation of the shallow water equations with topography by finite volume method, in a one-dimensional framework. Several single step methods have been derived from this formulation and numerical results were compared with the fractional steps method. Dellar and Salmon (2005) derived an extended set of shallow water equations that describes a thin inviscid fluid layer above fixed topography in a frame rotating about an arbitrary axis. Generalization of classical shallow water theory to the case of flows over an irregular bottom was shown by Karelsky et al. (2000). They showed that the simple self-similar solutions that are characteristic for the classical problem exist only if the underlying surface has a uniform slope. George (20008) also presented a class of augmented approximate Riemann solvers for the one-dimensional shallow water equations in the presence of an irregular bottom, neglecting the effect of Coriolis force. These methods belong to the class of finite volume Godunov type methods that use a set of propagation jump discontinuities, or waves, to approximate the true Riemann solution.

The fractional steps technique was applied for the numerical solution of the shallow water equations with flat bottom in the presence of the Coriolis force, (Shourci,2004). The method of fractional steps that he presented in his paper has the great advantage of solving the shallow water equations without the iterative steps involved in the multi-dimensional interpolation, and without the iteration associated with the intermediate step of solving the Helmholtz equation (Stainiforth and Temperton, 1986).

Rotating shallow water equations including topographic terms are numerically dealt with by the fractional steps method. In most real applications there is variable bottom topography that adds a source term to the 
shallow water equations. There are several works, where both the Coriolis forces as well as the bottom topography are taken into account, (Talibi and Tber, 2004).

In our work, we considered the shallow water equations with source terms the varied bottom topography and the Coriolis forces in two dimensions which makes it different from the existing works in the literature. Therefore a mathematical model of geophysical fluid flow over variable bottom topography was derived in the presence of Coriolis force. A system of three nonlinear partial differential equations was formulated in two-dimensions including the Coriolis force and bottom topography. We thus tried to determine the different kind of flow patterns possible with geophysical fluid flow characteristics, investigate the causes as well as factors leading to the flow pattern in this fluid flow. The solution to the modelled equations was by Perturbation method. We finally illustrated graphically our results which helped us in the interpretation of the modeled equations with the results.

\section{Mathematical Formulation of the Problem Assumptions of the Model}

Here we make the following assumptions for the model:

1. for this geophysical fluid flows; the horizontal length scale (L) is much larger than the vertical length scale (h). On a large scale this implies that the flow is predominantly horizontal and the vertical acceleration is small compared to the gravity acceleration.

2. The Cartesian coordinates $x, y$ and $z$ will be used, with measured vertically upward.

3. The velocity components in the directions of increasing $x, y$ and $z$ will be denoted by $u, v$ and $w$

4. We take the $(y, x$,$) horizontal plane as$ being parallel to the surface of the still water, and the depth of the water at a given point as $h=(x ; y ; t)>0$.

5. We denote velocity in the $x$-direction as $u=u(x ; y ; t)=$ and the velocity in the $\mathrm{y}-$ direction as $v=v(x ; y ; t)$. While the plane $(z=0)$ can be chosen arbitrarily, it is usually positioned at mean water level.

6. Measuring down from this plane, the bottom of the flow field is at depth $z=-\zeta(x, y)$.

The equation $z=-\zeta(x, y)$ is the equation for the bottom surface, also known as the variable bottom topography, the depth of which is usually assumed to vary with $x$ and $y$.

\section{The Model}

We hereby derive the momentum and Continuity equation for the two-dimensional shallow water flow model taking into account the effects of topography and the Earth's rotation. Consider a block of water of width $x \delta$ and $y \delta$ in the $x$ - and $y$-direction respectively with the height $h(x, y, t)$ and the Fluid velocity $u(x, y, t)$ and $v(x, y, t)$ in the $x$ - and $y$-direction respectively as shown in Figure below,

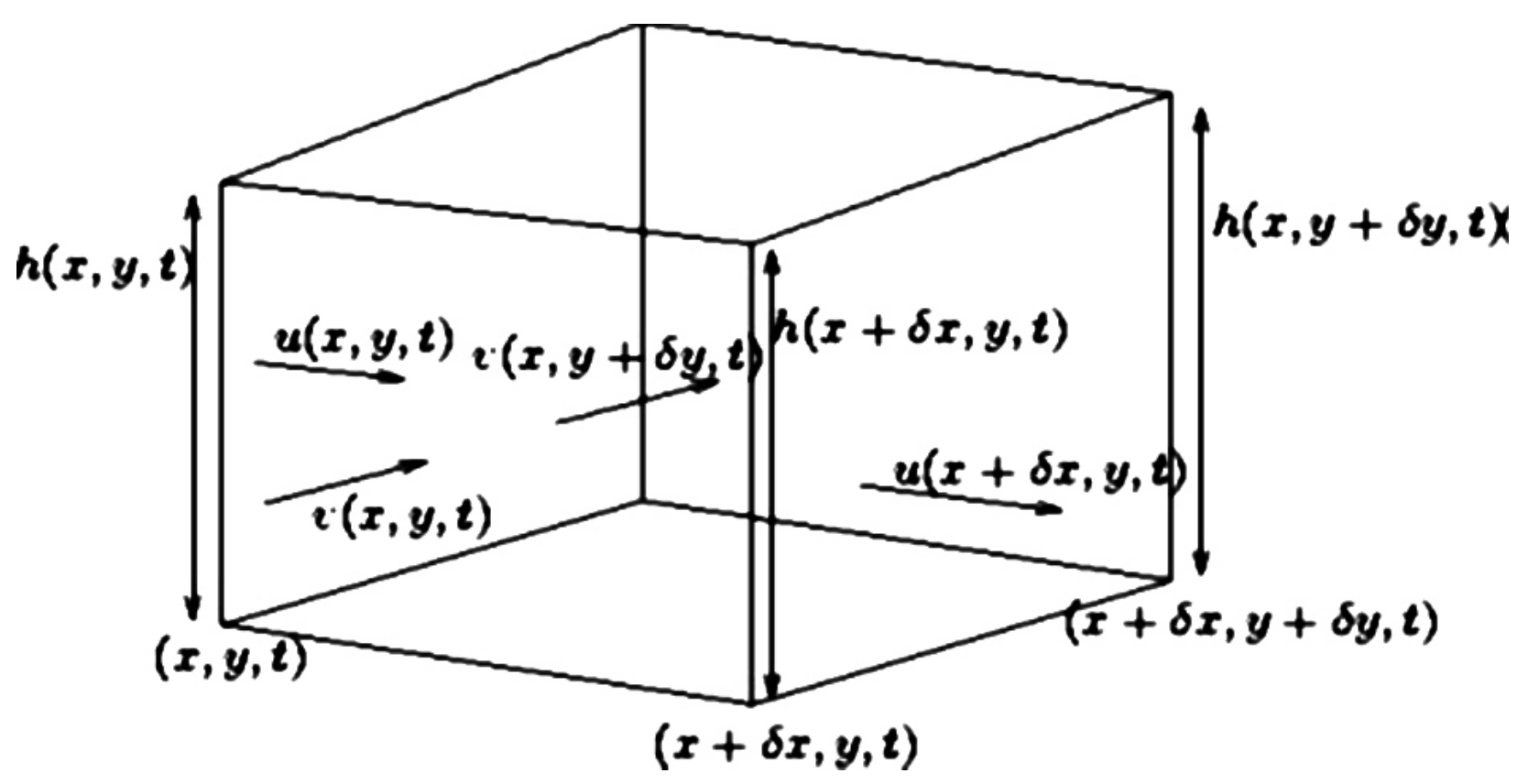

Figure 4.2: A block of fluid showing the velocities and heights 
By Newton's law of motion,

$F=m a$,

where $F$ is the sum of all forces, " $m$ " is mass, and " $a$ " is acceleration of the block of water.

\section{i) Change in momentum.}

Momentum $=q=$ mass $\times$ velocity, so let mass of the control volume be;

$$
\rho(u(x, y, t)+v(x, y, t)) h(x, y, t) \delta t
$$

and let velocity be $u(x, y, t)$, then change in momentum, per unit of time $\delta t$ is;

$$
\frac{\delta q}{\delta t}=\int_{x}^{x+\delta x} \frac{\partial}{\partial \hat{x}}\left(\rho u^{2}(\hat{x}, \hat{y}, t) h(\hat{x}, \hat{y}, t)\right) d \hat{x}+\int_{y}^{y+\delta y} \frac{\partial}{\partial \hat{y}}(\rho u(\hat{x}, \hat{y}, t) v(\hat{x}, \hat{y}, t) h(\hat{x}, \hat{y}, t)) d \hat{y}
$$

ii). Force due to the free surface of water is related to the potential energy of the wave which is given as,

$$
m g \eta=\rho h \delta x g(h+\xi)
$$

where $m=\rho h \delta x$ is the mass, $g$ is force due to gravity, $h=\frac{1}{2}(h(x, y, t)+h(x+\delta x, y, t))$ is the average height and $\eta=(h+\xi)$ is the wave amplitude. No w the change in energy over the control volume is due to changes in $\eta$, in other words, it is

$$
\begin{aligned}
& \delta(P E)=\operatorname{\rho gh} \delta x\{(h(x+\delta x, y, t)+\xi(x+\delta x, y))-(h(x, y, t)+\xi(x, y))\} \\
& \delta(P E)=\rho g h \delta x\{(h(x+\delta x, y, t)-h(x, y, t))+(\xi(x+\delta x, y)-\xi(x, y))\}
\end{aligned}
$$

So the force due to the free surface of the water is change in energy per change in distance. Therefore;

$$
\frac{\delta(P E)}{\delta x}=\int_{x}^{x+\delta x} \rho g h \frac{\partial}{\partial \hat{x}}(h(\hat{x}, y, t)+\xi(\hat{x}, y)) d \hat{x}
$$

iii). On the right hand side (RHS) of Newton's equation, the mass of the volume of water is $\rho \delta x h(x, y, t)$, and the acceleration is the derivative with respect to time of $u(x, y, t)$. The (RHS) expression is therefore

$$
\frac{\delta(m a)}{\delta x}=\frac{\partial}{\partial t} \int_{x}^{x+\delta x} \rho h(\hat{x}, y, t) u(\hat{x}, y, t) d \hat{x}=\int_{x}^{x+\delta x} \frac{\partial}{\partial t}(\rho u(\hat{x}, y, t) h(\hat{x}, y, t)) d \hat{x}
$$

Combining the integrals (1), (2) and (3) and taking the limit as $\delta x \rightarrow 0$, dividing by $\rho$, we arrive at the equation;

$$
\frac{\partial(h u)}{\partial t}+\frac{\partial\left(u^{2} h+g h^{2} / 2\right)}{\partial x}+\frac{\partial(h u v)}{\partial y}=-g h \frac{\partial \xi}{\partial x}
$$

The derivation for the y-component uses the same arguments, except that we now interchange $x$ with $y$ and $u$ with $y$. Alternatively, rotate the $(y, x)$ axis by $\frac{\pi}{2}$. Note that in performing this rotation of axes, the new axis will be pointing in the opposite direction from the direction of the original axis, but since $v$ was measured as positive in the original axis-direction, we change the sign of both and so the equation remains invariant.

Making the same analysis as for the $-x$ direction equation, we find the second equation, as

$$
\frac{\partial(h v)}{\partial t}+\frac{\partial(h u v)}{\partial x}+\frac{\partial\left(v^{2} h+\frac{g h^{2}}{2}\right)}{\partial y}=-g h \frac{\partial \xi}{\partial y}
$$

Let the force due to rotation in - and $y-$ be $(-f h v, f h u)$ where $f$ is the force due to rotation (Coriolis force) and is defined asb $f=2 \Omega \sin \varphi$

Therefore the momentum equation for the two dimensional directions are

$$
\frac{\partial(h u)}{\partial t}+\frac{\partial\left(u^{2} h+{ }^{g h^{2}} / 2\right)}{\partial x}+\frac{\partial(h u v)}{\partial y}=-g h \frac{\partial \xi}{\partial x}+f v
$$




$$
\frac{\partial(h v)}{\partial t}+\frac{\partial(h u v)}{\partial x}+\frac{\partial\left(v^{2} h+g h^{2} / 2\right)}{\partial y}=-g h \frac{\partial \xi}{\partial y}-f u
$$

\section{Continuity Equation}

Let us consider the fact that mass of fluid is conserved. Let the volume domain $A(t)$ be a column of water perpendicular to the $(x, y)$ plane, of height $h$ and small cross-sectional area $\partial A(t)$ at time $t$, which evolves to $\partial A(t+\delta t)$ at time $t+\delta t$. The column of water is moving with the flow, so in this sense the derivation can be considered to be following a Lagrangian co ordinate system. We require that the water mass of the column is conserved, which is equivalent to saying that the rate at which the mass changes are zero:

$$
\frac{D}{D t} \iint_{A(t)} \rho h d x d y=0
$$

That is rate of change of mass in column that moves with the flow, and $\rho$ is density of the substance.

Using the transport theorem, which state that,

$$
\frac{D}{D t} \iint_{A(t)} F(x, t) d V=\iint_{A(t)} \frac{D F}{D t}+F \nabla \cdot u d V
$$

We can then have

$$
\frac{D}{D t} \iint_{A(t)} \rho h d A=\iint_{A(t)} \frac{\partial(\rho h)}{\partial t} d A+\iint_{A(t)} \nabla \cdot(\rho h u) d A=0
$$

Using the divergence theorem, we then have:

$$
\iint_{A(t)} \frac{\partial(\rho h)}{\partial t} d A+\oint_{\partial A} \rho h u . \hat{n} d s=0
$$

Assuming continuity of the integrand, and that ${ }_{A}()$ is arbitrary, we can say that

$$
\begin{aligned}
& \frac{\partial(\rho h)}{\partial t}+\nabla \cdot(\rho h u)=0 \\
& \frac{\partial(\rho h)}{\partial t}+\frac{\partial(\rho h u)}{\partial x}+\frac{\partial(\rho h v)}{\partial y}=0
\end{aligned}
$$

and as $\rho$ is constant with time and also constant in the $x$-and $y$ - coordinates, it can be eliminated to give

$$
\frac{\partial h}{\partial t}+\frac{\partial(h u)}{\partial x}+\frac{\partial(h v)}{\partial y}=0
$$

The geophysical flows (shallow water) model over varied topography is complete hence the model equations are as follows;

$$
\left.\begin{array}{l}
\frac{\partial(h u)}{\partial t}+\frac{\partial\left(h u^{2}+g h^{2} / 2\right)}{\partial x}+\frac{\partial(h u v)}{\partial y}=-g \frac{\partial(\xi)}{\partial x}+f v \\
\frac{\partial(h v)}{\partial t}+\frac{\partial(h u v)}{\partial x}+\frac{\partial\left(h v^{2}+g h^{2} / 2\right)}{\partial y}=-g \frac{\partial(\xi)}{\partial y}-f u \\
\frac{\partial h}{\partial t}+\frac{\partial(h u)}{\partial x}+\frac{\partial(h v)}{\partial y}=0
\end{array}\right\}
$$

\section{Solution of the Problem}

We will consider the model equations

$$
\left.\begin{array}{ll}
\frac{\partial(h u)}{\partial t}+\frac{\partial\left(h u^{2}+g h^{2} / 2\right)}{\partial x}+\frac{\partial(h u v)}{\partial y}=-g \frac{\partial(\xi)}{\partial x}+f v & (a) \\
\frac{\partial(h v)}{\partial t}+\frac{\partial(h u v)}{\partial x}+\frac{\partial\left(h v^{2}+g h^{2} / 2\right)}{\partial y}=-g \frac{\partial(\xi)}{\partial y}-f u & (b) \\
\frac{\partial h}{\partial t}+\frac{\partial(h u)}{\partial x}+\frac{\partial(h v)}{\partial y}=0 & \text { (c) }
\end{array}\right\}
$$


with initial condition and boundary conditions:

$$
\left.\begin{array}{lll}
u(x, y, 0)=u_{0}, & u_{x}(0, y, t)=0, & u_{x}(l, y, t)=0 \\
v(x, y, 0)=v_{0}, & u_{y}(x, 0, t)=0, & u_{y}(x, n, t)=0 \\
v_{y}(x, 0, t)=0, & v_{y}(x, n, t)=0 \\
v_{x}(0, y, t)=0, & v_{x}(l, y, t)=0 \\
h(x, y, 0)=m e^{-s\left(x^{2}+y^{2}\right)}-\xi(x, y), & h_{x}(0, y, t)=0, h_{x}(l, y, t)=0 \\
h_{y}(x, 0, t)=0, & h_{y}(x, n, t)=0
\end{array}\right\}
$$

where

$$
\xi(x, y)=\alpha \sin (\beta x) 0 \leq \beta x \leq 90
$$

and the values of $\alpha$ and $\beta$ depends on the size of the hum

$$
h(x, y, t=0)=m e^{-s\left(x^{2}+y^{2}\right)}-\xi(x, y)
$$

for $l=\max x-$ axis and $n=\max y-$ axis and $g=10 \mathrm{~ms}^{-2}$

Equation $(c)$ in $(10)$ can be written as;

That is,

$$
\frac{\partial h}{\partial t}+h \frac{\partial u}{\partial x}+u \frac{\partial h}{\partial x}+h \frac{\partial v}{\partial y}+v \frac{\partial h}{\partial y}=0
$$

$$
\frac{\partial h}{\partial t}+u \frac{\partial h}{\partial x}+v \frac{\partial h}{\partial y}+h\left(\frac{\partial u}{\partial x}+\frac{\partial v}{\partial y}\right)=0
$$

This implies

$$
\frac{d h}{d t}+h\left(\frac{\partial u}{\partial x}+\frac{\partial v}{\partial y}\right)=0
$$

That is,

$$
\frac{d h}{d t}=-h\left(\frac{\partial u}{\partial x}+\frac{\partial v}{\partial y}\right)
$$

Equation $(a)$ in $(10)$ can be written as;

$$
\begin{aligned}
& \frac{\partial(h u)}{\partial t}+\frac{\partial\left(h u^{2}+g h^{2} / 2\right)}{\partial x}+\frac{\partial(h u v)}{\partial y}=-g \frac{\partial(\xi)}{\partial x}+f v \\
& u \frac{\partial h}{\partial t}+h \frac{\partial u}{\partial t}+u^{2} \frac{\partial h}{\partial x}+h u \frac{\partial u}{\partial x}+h \frac{\partial u^{2}}{\partial x}+g h \frac{\partial h}{\partial x}+h u \frac{\partial v}{\partial y}+h v \frac{\partial u}{\partial y}+u v \frac{\partial h}{\partial y}=-g \frac{\partial \xi}{\partial x}+f v \\
& u\left(\frac{\partial h}{\partial t}+u \frac{\partial h}{\partial x}+v \frac{\partial h}{\partial y}\right)+h\left(\frac{\partial u}{\partial t}+u \frac{\partial u}{\partial x}+v \frac{\partial u}{\partial y}\right)+h u\left(\frac{\partial u}{\partial x}+\frac{\partial v}{\partial y}\right)+g h \frac{\partial h}{\partial x}=-g \frac{\partial \xi}{\partial x}+f v
\end{aligned}
$$

Using (12) in (13), we obtain;

$$
h \frac{d u}{d t}+h g \frac{\partial h}{\partial x}=-g \frac{\partial \xi}{\partial x}+f v
$$

Similarly, equation $(b)$ in $(10)$ can be written as;

$$
v\left(\frac{\partial h}{\partial t}+u \frac{\partial h}{\partial x}+v \frac{\partial h}{\partial y}\right)+h\left(\frac{\partial v}{\partial t}+u \frac{\partial v}{\partial x}+v \frac{\partial v}{\partial y}\right)+h v\left(\frac{\partial u}{\partial x}+\frac{\partial v}{\partial y}\right)+g h \frac{\partial h}{\partial y}=-g \frac{\partial \xi}{\partial y}-f u
$$

Using (12) in (15), we obtain;

$$
h \frac{d v}{d t}+h g \frac{\partial h}{\partial y}=-g \frac{\partial \xi}{\partial y}-f u
$$

Now let $0<f<<1, \quad g=a f$.

We will solve this system by the perturbation method. To this end, suppose the solution $(u, v, h)$ can be expressed in series form as: 


$$
\left.\begin{array}{l}
u(x, y, t)=u_{0}(x, y, t)+f u_{1}(x, y, t)+\cdots \\
v(x, y, t)=v_{0}(x, y, t)+f v_{1}(x, y, t)+\cdots \\
h(x, y, t)=h_{0}(x, y, t)+f h_{1}(x, y, t)+\cdots
\end{array}\right\}
$$

Substituting equation (17) in (12), we have,

$$
\begin{aligned}
& \frac{d}{d t}\left(h_{0}+f h_{1}+\ldots\right)+\left(h_{0}+f h_{1}+\ldots\right)\left(\frac{\partial}{\partial x}\left(u_{0}+f u_{1}+\ldots\right)+\frac{\partial}{\partial y}\left(v_{0}+f v_{1}+\cdots\right)\right)=0 \\
& \left.\frac{d}{d t} h_{0}+\frac{d}{d t} f h_{1}+\ldots+h_{0} \frac{\partial}{\partial x} u_{0}+h_{0} \frac{\partial}{\partial x} f u_{1}+f h_{1} \frac{\partial}{\partial x} u_{0}+f^{2} h_{1} \frac{\partial}{\partial x} f u_{1}+\cdots+\right) \\
& h_{0} \frac{\partial}{\partial y} v_{0}+h_{0} \frac{\partial}{\partial y} f v_{1}+f h_{1} \frac{\partial}{\partial y} v_{0}+f^{2} h_{1} \frac{\partial}{\partial y} f v_{1}+\cdots=0
\end{aligned}
$$

Substituting equation (17) in (14), we have,

$$
\left.\begin{array}{l}
\left(h_{0}+f h_{1}+\cdots\right) \frac{d}{d t}\left(u_{0}+f u_{1}+\cdots\right)+a f\left(h_{0}+f h_{1}+\cdots\right) \frac{\partial}{\partial x}\left(h_{0}+f h_{1}+\cdots\right)=-a f \frac{\partial \xi}{\partial x}+f\left(v_{0}+f v_{1}+\cdot\right. \\
h_{0} \frac{d}{d t} u_{0}+f h_{0} \frac{d}{d t} u_{1}+f h_{1} \frac{d}{d t} u_{0}+f^{2} h_{1} \frac{d}{d t} u_{1}+\cdots+a f h_{0} \frac{\partial}{\partial x} h_{0}+a f^{2} h_{0} \frac{\partial}{\partial x} h_{1}+ \\
a f^{2} h_{1} \frac{\partial}{\partial x} h_{0}+a f^{3} h_{1} \frac{\partial}{\partial x} h_{1}+\cdots+=-a f \frac{\partial \xi}{\partial x}+f v_{0}+f^{2} v_{1}+\cdots
\end{array}\right\}
$$

Substituting equation (17) in (16), we have,

$$
\left.\begin{array}{l}
\left(h_{0}+f h_{1}+\cdots\right) \frac{d}{d t}\left(v_{0}+f v_{1}+\cdots\right)+a f\left(h_{0}+f h_{1}+\cdots\right) \frac{\partial}{\partial y}\left(h_{0}+f h_{1}+\cdots\right)=-a f \frac{\partial \xi}{\partial y}+f\left(u_{0}+f u_{1}+\cdots\right. \\
h_{0} \frac{d}{d t} v_{0}+f h_{0} \frac{d}{d t} v_{1}+f h_{1} \frac{d}{d t} v_{0}+f^{2} h_{1} \frac{d}{d t} v_{1}+\cdots+a f h_{0} \frac{\partial}{\partial y} h_{0}+a f^{2} h_{0} \frac{\partial}{\partial y} h_{1}+ \\
a f^{2} h_{1} \frac{\partial}{\partial y} h_{0}+a f^{3} h_{1} \frac{\partial}{\partial y} h_{1}+\cdots+=-a f \frac{\partial \xi}{\partial y}+f u_{0}+f^{2} u_{1}+\cdots
\end{array}\right\}
$$

Comparing the coefficient of powers of $f$ we have;

$f^{0}$ :

$$
\begin{aligned}
& h_{0} \frac{d u_{0}}{d t}=0 \quad ; \quad u_{0}(x, y, 0)=u_{0} \\
& h_{0} \frac{d v_{0}}{d t}=0 \quad ; \quad v_{0}(x, y, 0)=v_{0} \\
& \frac{d h_{0}}{d t}=-h_{0}\left(\frac{\partial u_{0}}{\partial x}+\frac{\partial v_{0}}{\partial y}\right) \quad ; \quad h_{0}(x, y, 0)=m e^{-s\left(x^{2}+y^{2}\right)}-\xi(x, y)
\end{aligned}
$$

$f^{1}:$

$$
\begin{gathered}
h_{0} \frac{d u_{1}}{d t}+h_{1} \frac{d u_{0}}{d t}+a h_{0} \frac{\partial h_{0}}{\partial x}=-a \frac{\partial \xi}{\partial x}+v_{0} ; \quad u_{1}(x, y, 0)=0 \\
h_{0} \frac{d v_{1}}{d t}+h_{1} \frac{d v_{0}}{d t}+a h_{0} \frac{\partial h_{0}}{\partial y}=-a \frac{\partial \xi}{\partial y}-u_{0} ; v_{1}(x, y, 0)=0 \\
\frac{d h_{1}}{d t}=-h_{1}\left(\frac{\partial u_{0}}{\partial x}+\frac{\partial v_{0}}{\partial y}\right)-h_{0}\left(\frac{\partial u_{1}}{\partial x}+\frac{\partial v_{1}}{\partial y}\right) ; h_{1}(x, y, 0)=0
\end{gathered}
$$

Integrating (21) with respect to $t$ we have;

$$
\begin{aligned}
& u_{0}(x, y, t)=C_{1}=\text { cons } \tan t \\
& u_{0}(x, y, 0)=C_{1}=u_{0}
\end{aligned}
$$


$\therefore \quad u_{0}(x, y, t)=u_{0}$

Integrating (22) with respect to $t$ we have;

$$
\begin{aligned}
& v_{0}(x, y, t)=C_{2}=\text { cons } \tan t \\
& v_{0}(x, y, 0)=C_{2}=v_{0} \\
\therefore \quad & v_{0}(x, y, t)=v_{0}
\end{aligned}
$$

Using (27) and (28), equation (23) reduces to

$$
\begin{aligned}
& \frac{d h_{0}}{d t}=-h_{0}(0+0)=0 \\
& \frac{d h_{0}}{d t}=0, h_{0}(x, y, 0)=d-\xi(x, y)=m e^{-s\left(x^{2}+y^{2}\right)}-\alpha \sin (\beta x)
\end{aligned}
$$

Integrating with respect to $t$, we have

$$
\begin{aligned}
& h_{0}(x, y, t)=C_{3}=\text { cons } \tan t \\
& h_{0}(x, y, 0)=C_{3}=m e^{-s\left(x^{2}+y^{2}\right)}-\xi(x, y) \\
& h_{0}(x, y, t)=m e^{-s\left(x^{2}+y^{2}\right)}-\alpha \sin (\beta x)
\end{aligned}
$$

Using (27), (28) and (29), equation (24) reduces to

$$
\begin{aligned}
& \frac{d u_{1}}{d t}=\frac{-a h_{0} \frac{\partial h_{0}}{\partial x}-a \frac{\partial \xi}{\partial x}+v_{0}}{h_{0}}=-a \frac{\partial h_{0}}{\partial x}-\frac{a}{h_{0}} \frac{\partial \xi}{\partial x}+\frac{v_{0}}{h_{0}} \\
& \frac{d u_{1}}{d t}=2 a m s x e^{-5\left(x^{2}+y^{2}\right)}+a \alpha \beta \cos \beta x-\frac{a \alpha \beta \cos \beta x}{m e^{-s\left(x^{2}+y^{2}\right)}-\alpha \sin \beta x}+\frac{v_{0}}{m e^{-s\left(x^{2}+y^{2}\right)}-\alpha \sin \beta x}
\end{aligned}
$$

Integrating with respect to $t$, we have

$$
u_{1}=\left(2 a m s x e^{-5\left(x^{2}+y^{2}\right)}+a \alpha \beta \cos \beta x-\frac{a \alpha \beta \cos \beta x}{m e^{-s\left(x^{2}+y^{2}\right)}-\alpha \sin \beta x}+\frac{v_{0}}{m e^{-s\left(x^{2}+y^{2}\right)}-\alpha \sin \beta x}\right) t+C 4
$$

But $u_{1}(x, y, 0)=0 \Rightarrow C_{4}=0$

$$
u_{1}(x, y, t)=\left(2 a m s x e^{-s\left(x^{2}+y^{2}\right)}+a \alpha \beta \cos \beta x-\frac{a \alpha \beta \cos \beta x}{m e^{-s\left(x^{2}+y^{2}\right)}-\alpha \sin \beta x}+\frac{v_{0}}{m e^{-s\left(x^{2}+y^{2}\right)}-\alpha \sin \beta x}\right) \times t
$$

Using (27), (28) and (29), equation (25) reduces to

$$
\begin{aligned}
& \frac{d v_{1}}{d t}=\frac{1}{h_{0}}\left(-a h_{0} \frac{\partial h_{0}}{\partial y}-a \frac{\partial \xi}{\partial y}-u_{0}\right) \\
& \frac{d v_{1}}{d t}=-a \frac{\partial h_{0}}{\partial y}-\frac{a}{h_{0}} \frac{\partial \xi}{\partial y}-\frac{u_{0}}{h_{0}} \\
& \frac{d v_{1}}{d t}=2 a m s y e^{-s\left(x^{2}+y^{2}\right)}-\frac{u_{0}}{m e^{-s\left(x^{2}+y^{2}\right)}-\alpha \sin \beta x}
\end{aligned}
$$

Integrating with respect to $t$, we have

$$
v_{1}=\left(2 a m s y e^{-s\left(x^{2}+y^{2}\right)}-\frac{u_{0}}{m e^{-s\left(x^{2}+y^{2}\right)}-\alpha \sin \beta x}\right) t+C 5
$$

But $v_{1}(x, y, 0)=0 \Rightarrow C_{5}=0$

$$
v_{1}(x, y, t)=\left(2 \operatorname{amsye} e^{-s\left(x^{2}+y^{2}\right)}-\frac{u_{0}}{m e^{-s\left(x^{2}+y^{2}\right)}-\alpha \sin \beta x}\right) t
$$

Now, using (27), (28), (29), (30) and (31)in equation (26) reduces to

$$
\frac{d h_{1}}{d t}=-h_{0}\left(\frac{\partial u_{1}}{\partial x}+\frac{\partial v_{1}}{\partial y}\right)-h_{1}\left(\frac{\partial u_{0}}{\partial x}+\frac{\partial v_{0}}{\partial y}\right)
$$


That is

$$
\frac{d h_{1}}{d t}=-h_{0}\left(\frac{\partial u_{1}}{\partial x}+\frac{\partial v_{1}}{\partial y}\right)
$$

This implies that,

$$
\begin{aligned}
& \frac{\partial u_{1}}{\partial x}=\left(\begin{array}{l}
2 a m s e^{-s\left(x^{2}+y^{2}\right)}-4 a m s^{2} x^{2} e^{-5\left(x^{2}+y^{2}\right)}+\alpha \beta^{2} \sin (\beta x)+\frac{a \alpha \beta^{2} \sin (\beta x)}{m e^{-s\left(x^{2}+y^{2}\right)}-\alpha \sin (\beta x)} \\
+\frac{a \alpha \beta \cos (\beta x)\left(-2 m s x e^{-s\left(x^{2}+y^{2}\right)}-\alpha \beta \cos (\beta x)\right) v_{0}\left(2 m s x e^{-s\left(x^{2}+y^{2}\right)}-\alpha \beta \cos (\beta x)\right)}{\left(m e^{-s\left(x^{2}+y^{2}\right)}-\alpha \sin (\beta x)\right)^{2}}\left(m e^{-s\left(x^{2}+y^{2}\right)}-\alpha \sin (\beta x)\right)^{2}
\end{array}\right) \times t \\
& \frac{\partial v_{1}}{\partial y}=2 a m s e^{-s\left(x^{2}+y^{2}\right)}-2 a m s^{2} y^{2} e^{-s\left(x^{2}+y^{2}\right)} \frac{2 m s u_{0} y e^{-s\left(x^{2}+y^{2}\right)}}{\left(m e^{-s\left(x^{2}+y^{2}\right)}-\alpha \sin (\beta x)\right)^{2}}
\end{aligned}
$$

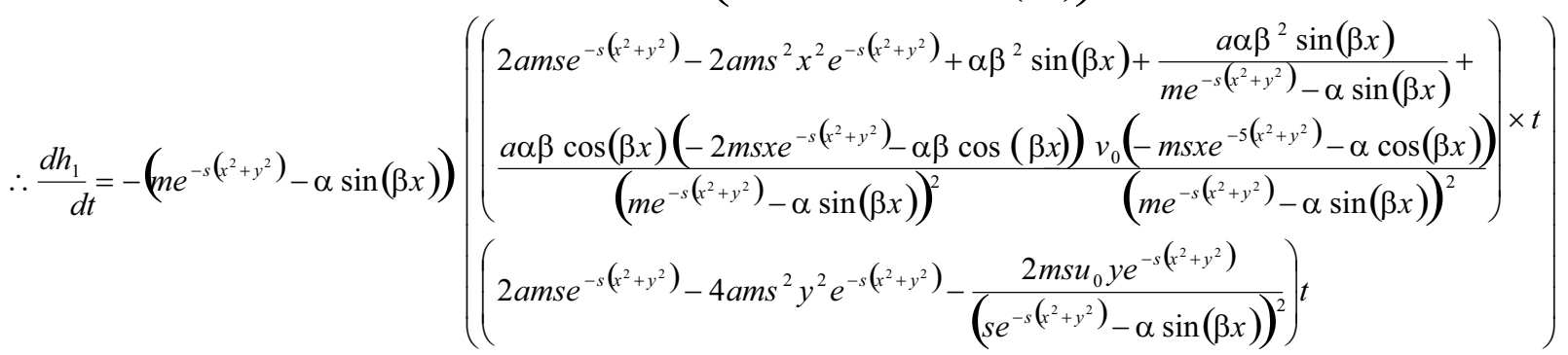

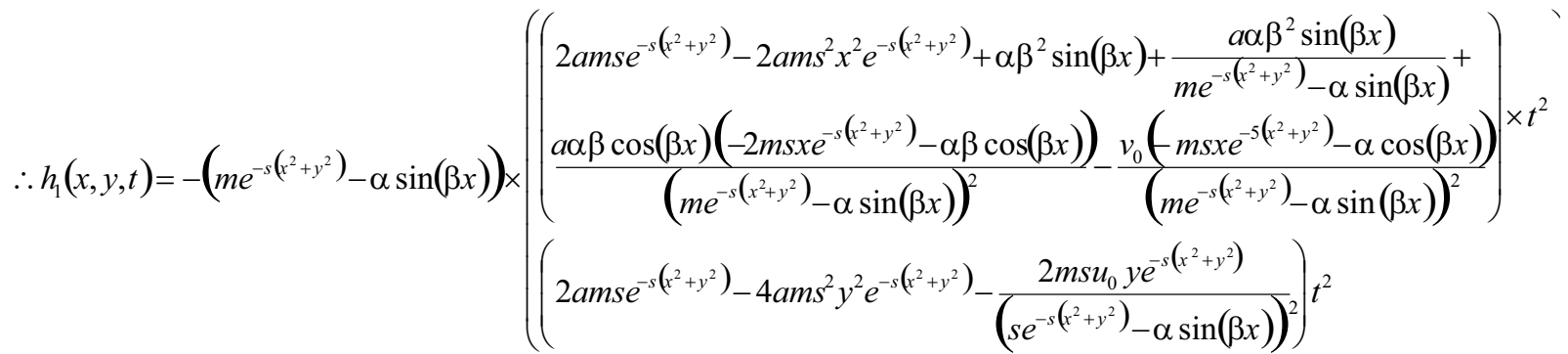

but $h_{1}(x, y, 0)=0 \Rightarrow C_{6}=0$

$\therefore h_{1}(x, y, t)=\left(\alpha \sin (\beta x)+m e^{-s\left(x^{2}+y^{2}\right)}\right) \times$

$$
\left(\begin{array}{l}
4 a m s e^{-s\left(x^{2}+y^{2}\right)}-2 a m s^{2} x^{2} e^{-s\left(x^{2}+y^{2}\right)}+\alpha \beta^{2} \sin (\beta x)+\frac{a \alpha \beta^{2} \sin (\beta x)}{m e^{-s\left(x^{2}+y^{2}\right)}-\alpha \sin (\beta x)}+ \\
\frac{a \alpha \beta \cos (\beta x)\left(-2 m s x e^{-s\left(x^{2}+y^{2}\right)}-\alpha \beta \cos (\beta x)\right)}{\left(m e^{-s\left(x^{2}+y^{2}\right)}-\alpha \sin (\beta x)\right)^{2}}-\frac{v_{0}\left(-m s x e^{-5\left(x^{2}+y^{2}\right)}-\alpha \cos (\beta x)\right)}{\left(m e^{-s\left(x^{2}+y^{2}\right)}-\alpha \sin (\beta x)\right)^{2}} \\
4 a m s^{2} y^{2} e^{-s\left(x^{2}+y^{2}\right)}-\frac{2 m s u_{0} y e^{-s\left(x^{2}+y^{2}\right)}}{\left(m e^{-s\left(x^{2}+y^{2}\right)}-\alpha \sin (\beta x)\right)^{2}}
\end{array}\right)+\frac{t^{2}}{2}
$$

Therefore;

$$
\begin{gathered}
u(x, y, t)=u_{0}(x, y, t)+f u_{1}(x, y, t)+\ldots \\
u(x, y, t)=u_{0}+\left(\begin{array}{l}
2 g m s x e^{-s\left(x^{2}+y^{2}\right)}+g \alpha \beta \cos (\beta x)-\frac{g \alpha \beta \cos (\beta x)}{\left(m e^{-s\left(x^{2}+y^{2}\right)}-\alpha \sin (\beta)\right)} \\
\frac{f v_{0}}{m e^{-s\left(x^{2}+y^{2}\right)}-\alpha \sin (\beta)}
\end{array}\right) t
\end{gathered}
$$

\section{Results and Discussion}

To be able to discuss the model equations and their solutions, we need a graphical sketch of what obtains with the type of the model and their solutions. Thus, using Maple software, we obtain the following results:

In Figure 1, when $1.0=f$ (that is the force 
due to rotation), the flow velocity is slowly varying with time. At $5.0=f$ the flow velocity increases rapidly as time increases and this is faster as compared to . Furthermore, at $1=f$ the flow velocity is high and increases with time at a greater rate than both $1.0=f$ and $5.0=f$. This shows that if the force due to rotation is high, the rate of flow or flow velocity is also high with increasing time.

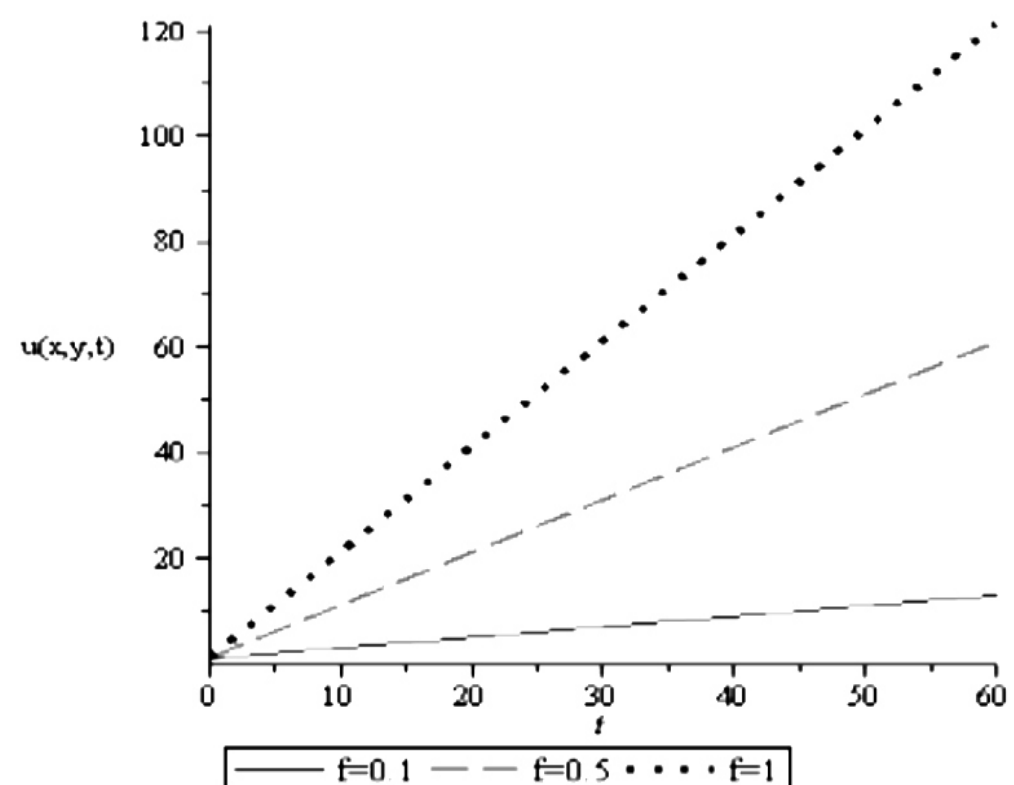

figure 1 variation of velocity $u(x, y, t)$ with time $t$, with different values of f.

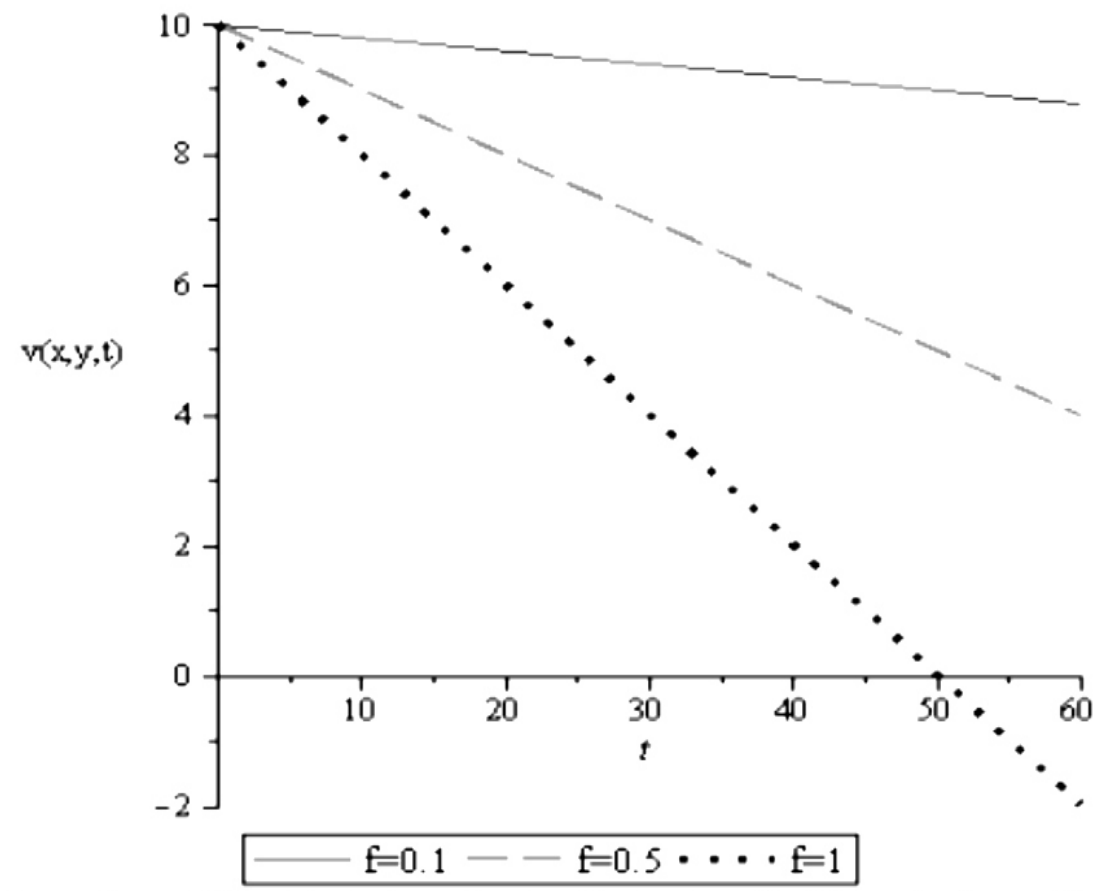

figure 2: variation of velocity $v(x, y, t)$ with time $t$, with different values of

Figure 2 is the graph of flow velocity $v(x, y, t)$ in the horizontal $-y$ direction with time $t$. This shows that the velocity is decreasing as time increases until it becomes zero. For $1.0=f$ the variation of the flow velocity is slow as compared to $5.0=f$ and $1=f$. This indicates that for increase in the values of the force due to rotation, the variation in flow velocity in the horizontal $-y$ direction will decrease at a faster rate and gradually turn to zero.

Figures 3 and 4 illustrates simple harmonic motion with different amplitudes. The 
flow is also turbulent giving fluctuations in velocities. Velocities are varying in space due to turbulent fluctuations in the force and we can see the variation in the velocity fluctuations with different levels of turbulence. In figure 3, the effect of forces due to rotation on the velocity $u(x, y, t)$ in the horizontal $-x$ direction, that is $1.0=f, 5.0=f$ and $1=f$ are the same giving resulting to the same fluctuations and turbulence. Figure 4 shows the flow velocity $v(x, y, t)$ in the horizontal $-y$ direction which shows that the level of turbulence is experienced more at and. This means that when the force due to rotation is high there will be high and different levels of disorderliness which is the turbulent fluctuations.
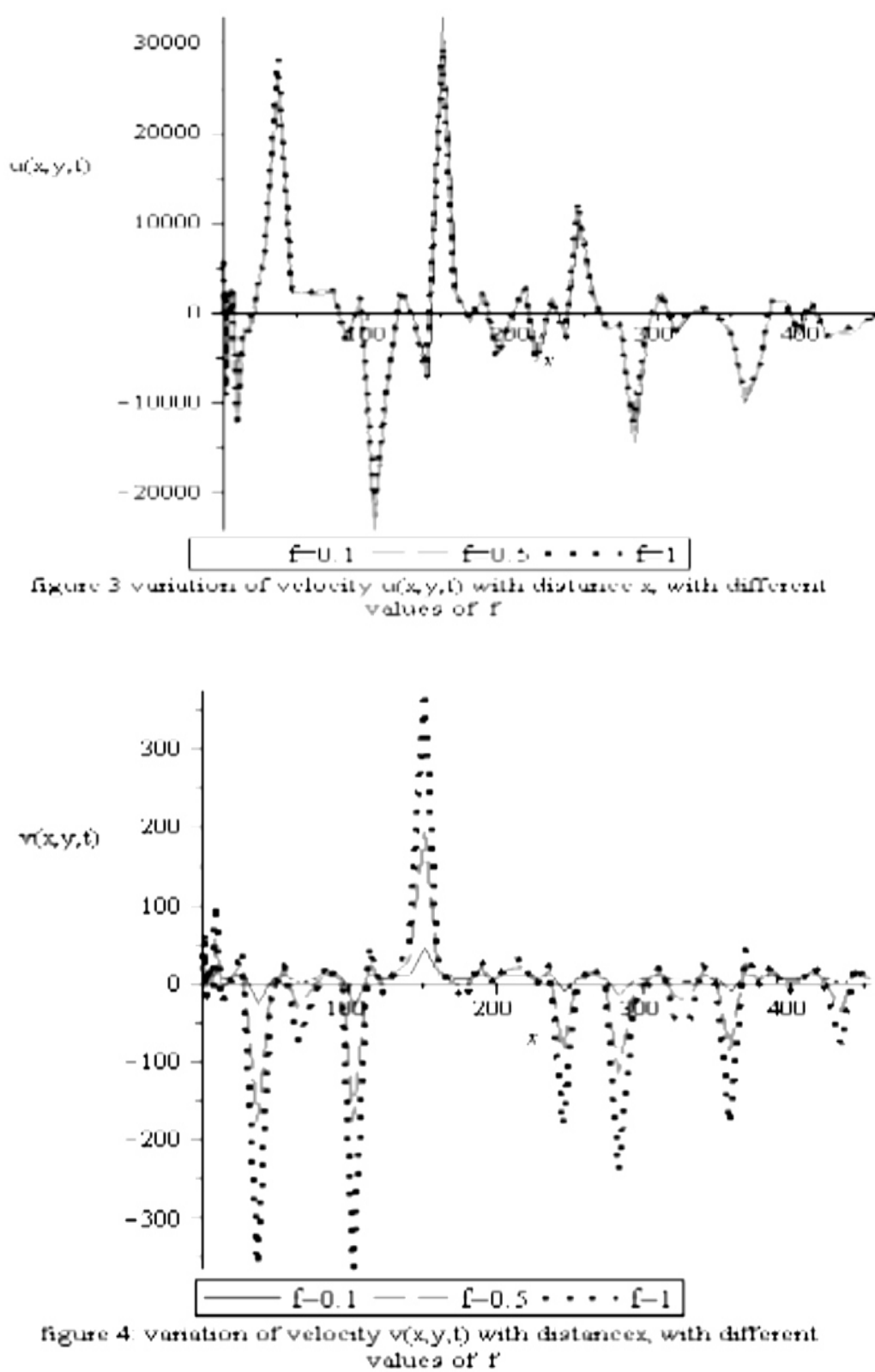

Figures 5 and 6 shows that the flow velocity $u(x, y, t)$ and $v(x, y, t)$ in the horizontal $y-$ direction are constant. For $1.0=f$ in figure 5 , the flow velocity has a constant motion of less than $10 \mathrm{~ms}^{-1}$ at each point, while $5.0=f$ and $1=f$ has $60 \mathrm{~ms}^{-1}$ and $120 \mathrm{~ms}^{-1}$ respectively. In the case of figure 6 the flow velocity $v(x, y, t)$ shows that when the force due to rotation is small, the flow velocity is high $\left(9 m s^{-1}\right)$ and so, at higher values, say at and the flow becomes imaginary. This indicates that irrespective of and, the flow velocity does not change from point to point at any instant of time due to change in the force of rotation. 


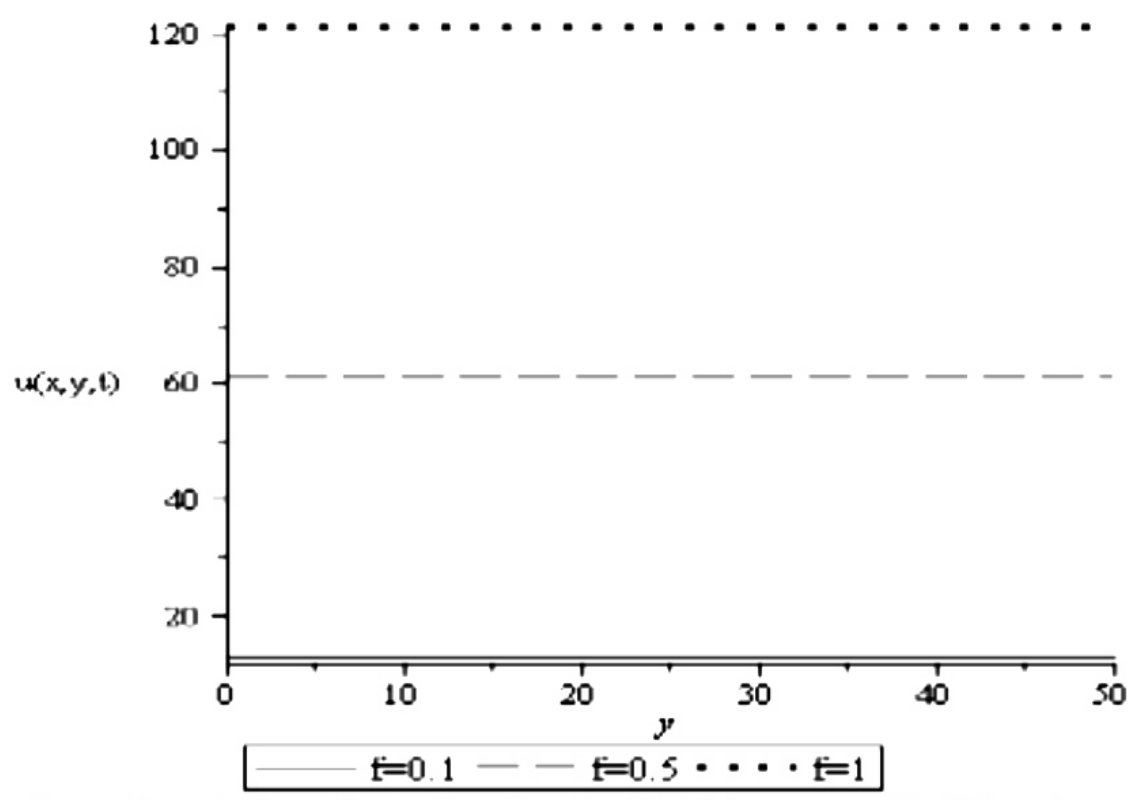

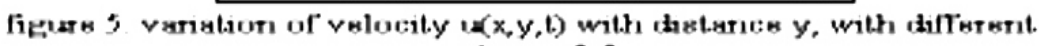
values of $f$

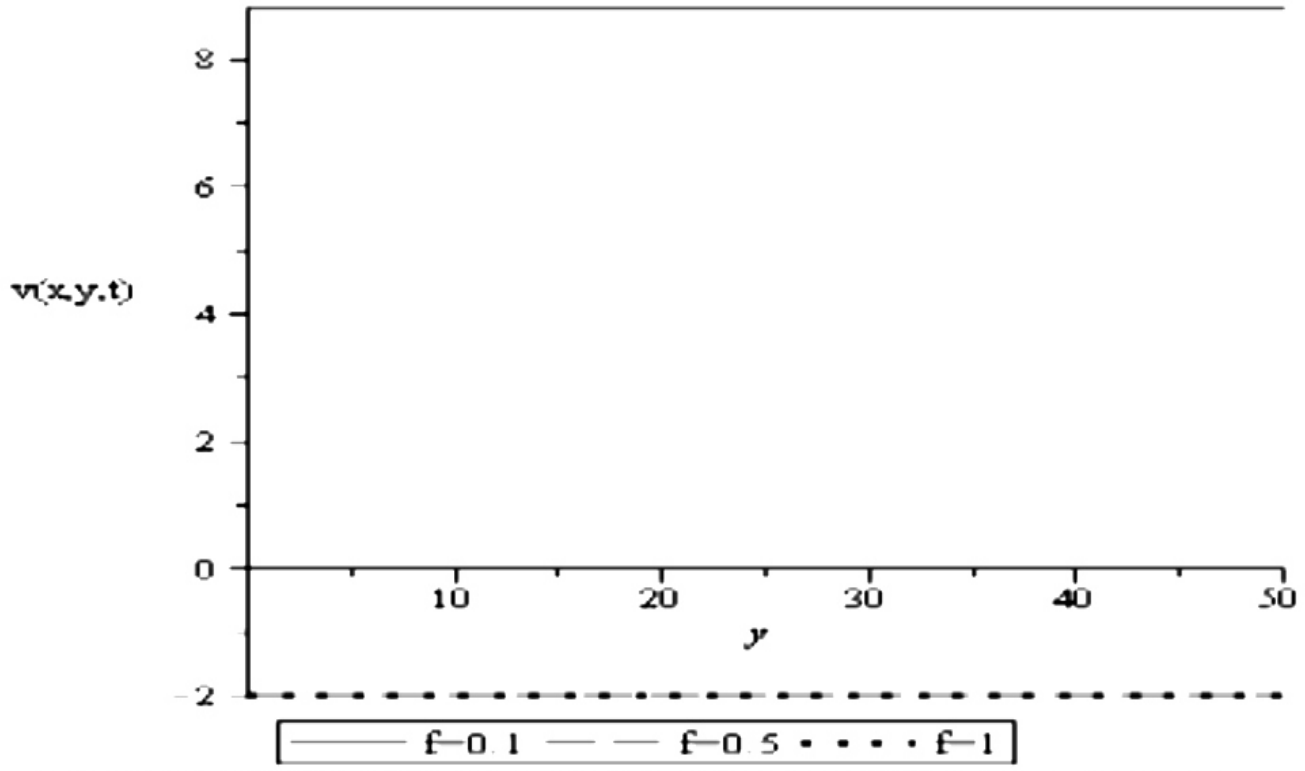
figure 6 : variation of velocity $v(x, y, t)$ with distance $y$. with different

Figures 7 and 8 shows the view of the variation of flow velocity $u(x, y, t)$ and $v(x, y, t)$ respectively which gives the plane shape that describes the surface flow velocity in $x$ direction with time $t$. The flow velocity is non-uniform at

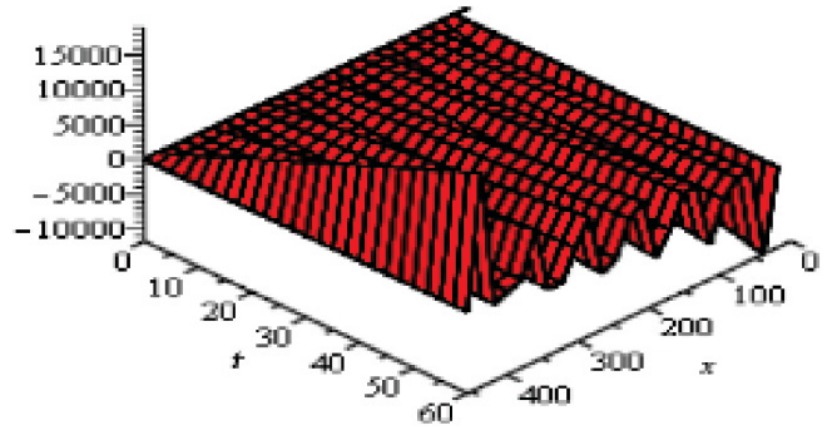

Figure 7: Variation of velocity $u(x, y, t)$ with horizontal distance $-x$ and time $t$ for different values of $f$ different times and space with the flow boundary freely deformable. At this point, overturning turbulence is generated resulting to mixing. Different colours indicate different forces due to rotation.

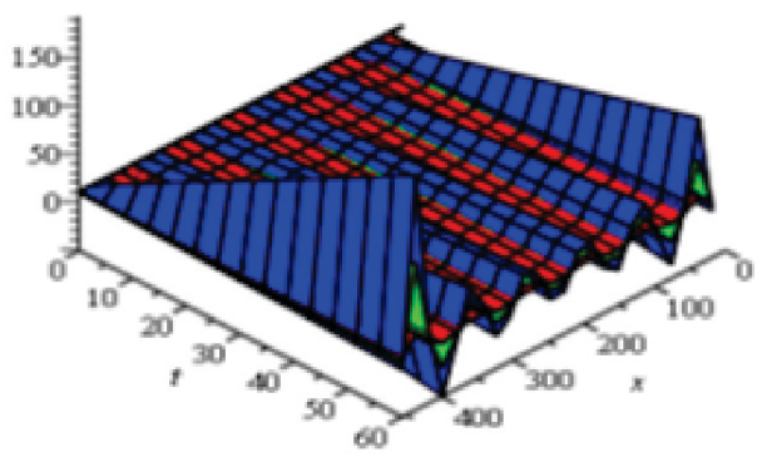

Figure 8: Variation of velocity

$v(x, y, t)$ with horizontal distance $-x$ and time $t$ for different values of $f$ 
The Figures 9, 10, 11 and12 describe a flat surface flow of the water which can be extending infinitely in all directions. The peak will be decreasing and the distribution will be spreading wider at different times and space in two dimensions. The surface of the flow thus formed is called a free surface, because the flow boundary is freely deformable, in contrast to the solid boundaries. The boundary conditions at the free surface of an open flow are always that

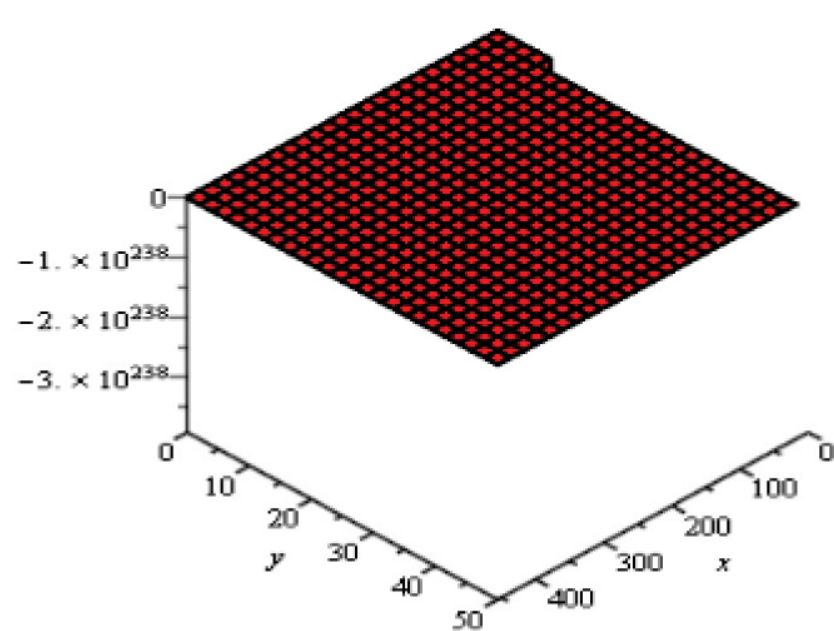

Figure 9: Variation of velocity $u(x, y, t)$ with horizontal distance - $y$ and time $t$ for different values of $f$

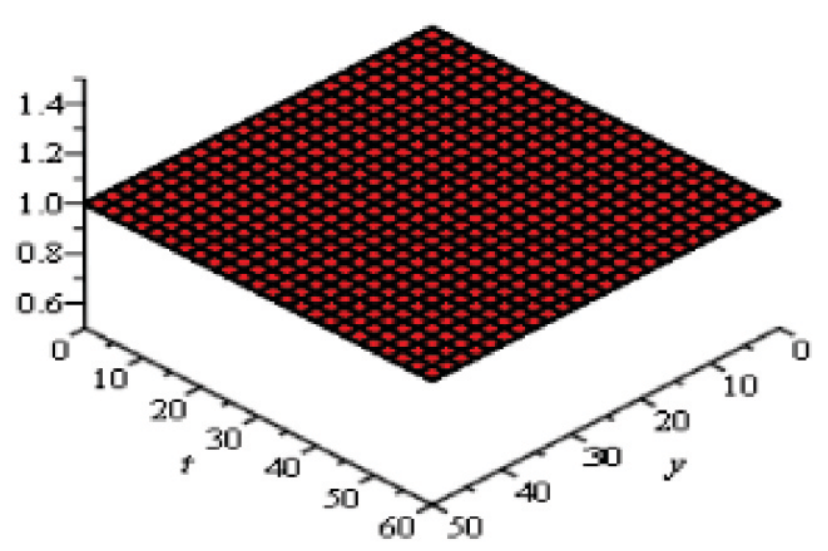

Figure 11: Variation of velocity $u(x, y, t)$ with horizontal distance - $y$ and time $t$ for different values of $f$

These results showed the dimensional arguments that the flow velocity in the horizontal directions varies at different points in time. It is a non-linear wave and the flow exhibited non-linearity, resulting in non-linear waves travelling continuously through the domain with vertical advective mixing because of overturning. The flow predicts nonlinear internal waves with overturning from unstable conditions due to the force of rotation and bottom topography. both the pressure and the shear stress are zero everywhere. Figures 9 and 11 shows specifically the view of the variation of flow velocity $u(x, y, t)$ and $v(x, y, t)$ with the horizontal distance $x$ and $y$ while Figures 10 and 12 shows the graph of the velocities and with the horizontal distance $y$ and time $t$. Different planes in Figure 12 are indicating different forces due to rotation (i.e $f=$ $0.1, f=0.5, f=1)$

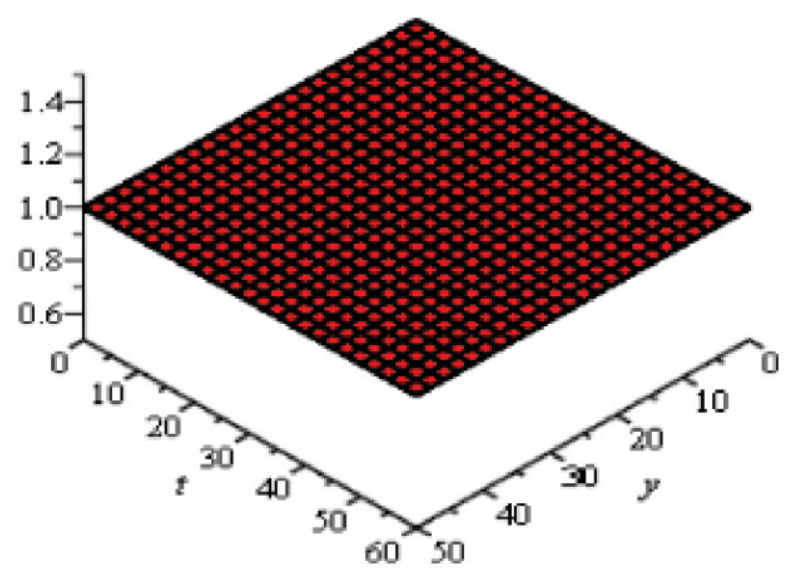

Figure 10: Variation of velocity

$u(x, y, t)$ with horizontal distance - $y$ and time $t$ for different values of $f$

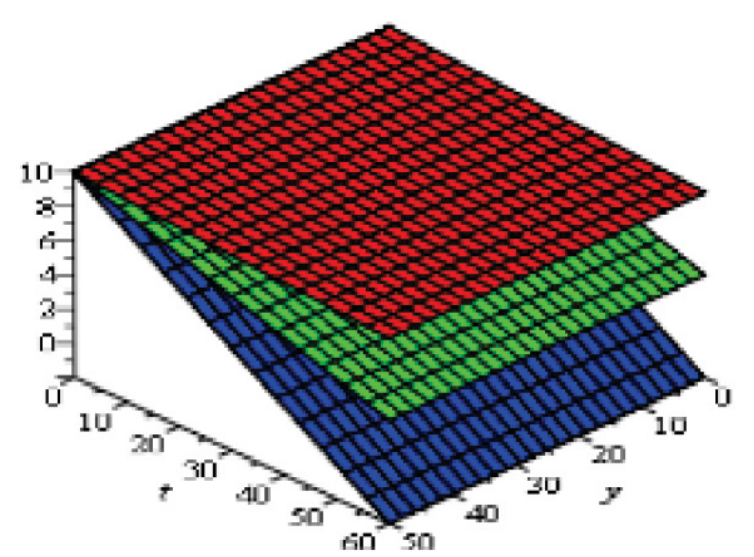

Figure 12: Variation of velocity

$v(x, y, t)$ with horizontal distance $-y$ and time $t$ for different values of $f$

\section{Conclusions}

In this work, we obtained the mathematical model of geophysical fluid flow over variable bottom topography. We applied perturbation method to solve the system of the three partial differential equations in twodimensions with Coriolis force and bottom topography included. The presence of the Coriolis force in the shallow water equations causes the deflection of fluid parcels in the direction of wave motion and causes gravity 
waves to disperse. As water depth decreases due to bottom topography, the wave amplitude increases, the wavelength and wave speed decreases resulting in overturning of the wave. The Coriolis force is responsible for the oscillatory motion in the direction of wave motion which causes gravity waves to disperse.

\section{References}

Dellar, P. J. and Salmon, R. (2005). Shallow Water Equations with a Complete Coriolis Force and Topography, Physics of Fluids, Vol. 17, No. 10, 106601106619.

Dritschel, D. G., Polvani, L. M. and Mohebalhojeh, A. R. (1999). The Contour-Advective Semi-Lagrangian Algorithm for the Shallow Water Equations, Monthly Weather Review, Vol. 127, No. 7, 1551-1564.

Gallouët, T., Hérard, J. M. and Seguin, N. (2003). Some Approximate Godunov Schemes to Compute Shallow-Water Equations with Topography, Computers \& Fluids, Vol. 32, No. 4, 479-513.

George, D. L. (2008). Augmented Riemann Solvers for the Shallow Water Equations over Variable Topography with Steady States and Inundation, Journal of Computational Physics, Vol. 227, No. 6, 3089-3113.
Karelsky, K. V., Papkov, V. V., Petrosyan, A. S. and Tsygankov, D. V. (2000). "Particular Solution of the ShallowWater Equations over a Non-Flat Surface, Physics Letters A, Vol. 271, No. 5-6, 341-348.

Lukácová-Medvid'ová, M., Noelle, S. and Kraft,M. (2007). Well- Balanced Finite Volume Evolution Galerkin Methods for the Shallow Water Equations," Journal of Computational Physics, Vol. 221, No. 1, 2007, 122-147.

Pedlosky, J. (1987) Geophysical Fluid Dynamics, Springer, New York.

Shoucri, M. (2004). The Application of a Fractional Steps Method for the Numerical Solution of the Shallow Water Equations, Computer Physics Communications, Vol. 164, No. 1-3, 396-401.

Stainiforth, A. and Temperton, C. (1986). SemiImplicit Semi- Lagrangian Integration Scheme for a Baratropic FiniteElement Regional Model, Monthly Weather Review, Vol. 114, No. 11, 1986, 2078-2090.

Talibi, M. E. and Tber, M. H. (2004). On a Problem of Shallow Water Type, Electronic Journal of Differential Equations, Vol. 11, 109-116. 